\title{
ANGIOMIOLIPOMA RENAL GIGANTE DETECTADO POR LEVE TRAUMATISMO LUMBAR Y TRATADO POR NEFRECTOMÍA: PRESENTACIÓN DE UN CASO
}

\author{
M. ROSSELLÓ BARBARÁ, A. MUS MALLEU, M. REBASSA LLUL, J. CASALS ARMADA \\ Centro de Urología, Andrología y Sexología de Baleares. Clínica Rotger. Palma de Mallorca (Baleares).
}

Actas Urol Esp. 28 (2): 133-137, 2004

\section{RESUMEN}

ANGIOMIOLIPOMA RENAL GIGANTE DETECTADO POR LEVE TRAUMATISMO LUMBAR Y TRATADO POR NEFRECTOMÍA: PRESENTACIÓN DE UN CASO

Se presenta un caso de angiomiolipoma renal gigante descubierto por el estudio de un leve traumatismo lumbar. Se demuestran lesiones diseminadas de Esclerosis Tuberosa en: cerebro, cerebelo, hígado y riñón contralateral. Externamente muestra estigmas cutáneos de esta enfermedad en la cara. El tamaño de la víscera y la pérdida de función del riñón izquierdo condicionan la nefrectomía. La pieza pesó al extraerse 6300 g, sin signos de malignización. Al examen microscópico se demostró esclerosis atrófica por compresión. Su evolución post-operatoria fue satisfactoria. Actualmente controla su tensión arterial con tratamiento médico.

PALABRAS CLAVE: Angiomiolipoma. Hamartoma. Esclerosis tuberosa. Enfermedad de Bourneville.

\section{ABSTRACT \\ GIANT ANGIOMYOLIPOMA OF THE KIDNEY DETECTED BY LUMBAR LIGHT TRAUMA AND TREATED BY NEPHRECTOMY: CASE REPORT}

A case of giant angiomyolipoma of the kidney discovered during the study of a light lumbar trauma is presented. Disseminated tuberous sclerosis lesions are demonstrated at the brain, cerebellum, liver and contralateral kidney. External cutaneous stigmas of this illness are shown at the face. The size of the viscera and the loss of function of the left kidney conditioned the nephrectomy. The piece weighed upon extracting $6300 \mathrm{~g}$, without malignant signs. To the microscopic exam atrophic sclerosis for compression was demonstrated. The postoperative course was satisfactory. Actually his arterial tension is controlled by medical treatment.

KEY WORDS: Angiomyolipoma. Hamartoma. Tuberous sclerosis. Bourneville's disease.

$\mathrm{E}_{\mathrm{pec}}^{\mathrm{l}}$ angiomiolipoma renal (AML) es un tumor poco común de carácter benigno ${ }^{1}$. Es un hamartoma: término utilizado por vez primera en 1904, derivado de la palabra griega "hamartaneim" que significa: errar ${ }^{2}$. En clara referencia a un error del organismo en la formación de sus tejidos. Está compuesto por tejido graso: maduro e inmaduro, vasos sanguíneos de paredes delgadas y elementos de musculatura lisa en proporción variable ${ }^{3}$. Hay dos variantes de angiomiolipoma: una asociada a las facomatosis. Enfermedades caracterizadas por hamartomas que involucran numerosos tejidos ${ }^{4}$, como: la Esclerosis Tuberosa o Enfermedad de Bourneville, la enfermedad de von Recklinghausen o neurofibromatosis y la enfermedad de von Hippel Lindau. Y la otra variante en la que el AML renal es una enfermedad aislada. El paciente que presentamos se inscribe en un AML renal dentro del cuadro clínico de la Esclerosis Tuberosa o Enfermedad de Bourneville. 


\section{CASO CLÍNICO}

Paciente: JGMJ de 40 años de edad, masculino, casado, es visto por el servicio de urgencias de la Clínica Rotger el 29 de diciembre de 1998, por sufrir traumatismo lumbar izquierdo en su trabajo. Aqueja discreto dolor en fosa lumbar izquierda y se constatan constantes vitales normales y estables. La ecografía confirma gran tumoración del riñón izquierdo de más de $30 \mathrm{~cm}$ de diámetro máximo, caracterizada como angiomiolipoma que ocupa todo este riñón y otras lesiones semejantes parenquimatosas que se distribuyen en el riñón derecho. Al examen físico evidenciamos unas tuberosidades faciales distribuidas en alas de mariposa sobre el labio superior e inferior que se clasifican y diagnostican como Esclerosis Tuberosa o Enfermedad de Bourneville (Fig. 1).

Es remitido al Centro de Urología 4 días más tarde habiendo cursado su historia clínica con fiebre de $38,5^{\circ} \mathrm{C}$, como manifestación de infección del tracto urinario que cedió con antibioticoterapia. Acude a consulta y en la exploración clínica le palpo gran masa de bordes imprecisos, discreta-

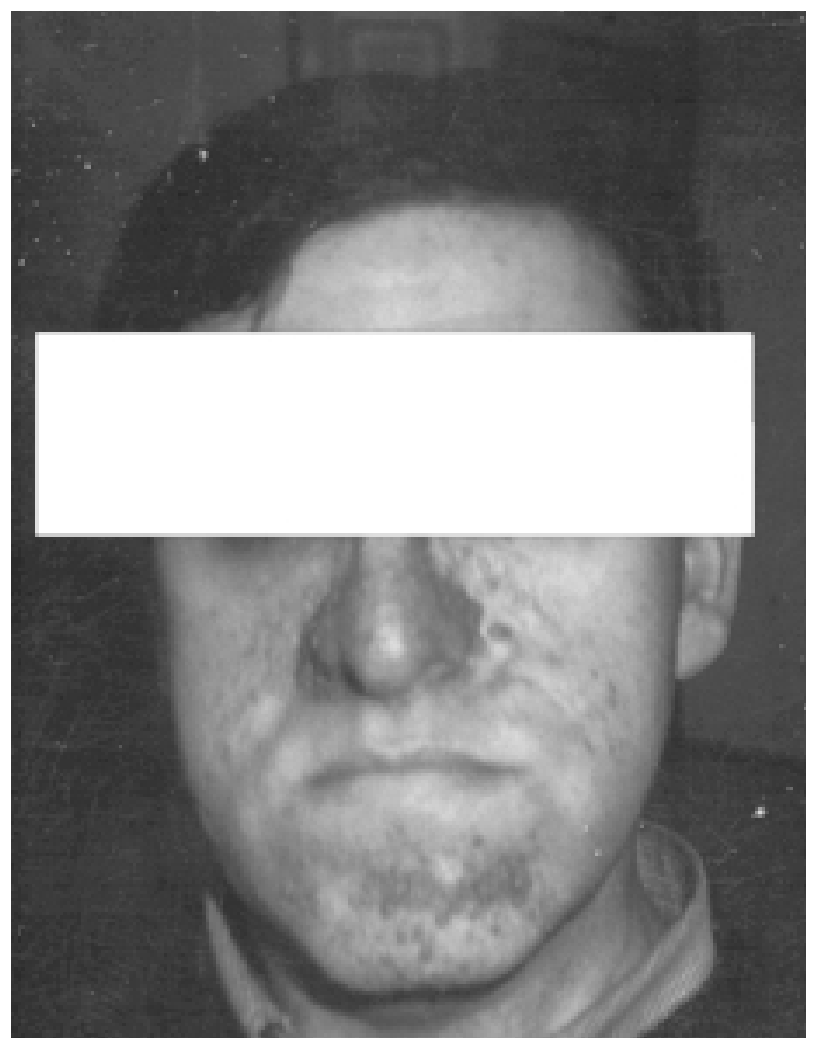

FIGURA 1. Aspecto de las lesiones tuberosas cutáneas de nuestro paciente. mente dolorosa a la presión profunda situada en región lumbar izquierda y vacío izquierdo, llegando a fosa ilíaca izquierda y sobrepasa la línea media abdominal. Se le pide un TAC, PIV y analítica completa. En el TAC del 30 de diciembre de 1998 se constata: a nivel del cráneo múltiples y pequeñas imágenes nodulares calcificadas de localización subependimaria, compatibles con hamartomas típicos de la esclerosis tuberosa (Fig. 2).

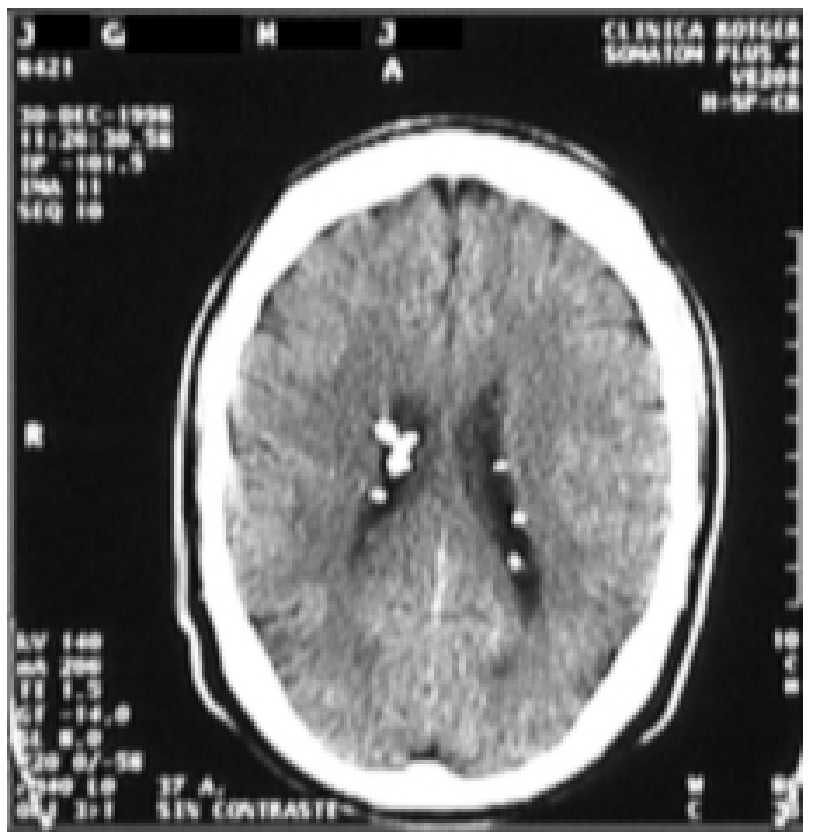

FIGURA 2. Hamartomas calcificados subependimarios.

Así como a nivel del hemisferio cerebeloso izquierdo también compatible con hamartomas calcificados corticales (Fig. 3).

A nivel abdominal el riñón derecho muestra múltiples imágenes nodulares de predominio cortical y contenido parcialmente graso compatibles con angiomiolipomas. El sistema excretor es normal. En el lado izquierdo se observa severo aumento de tamaño y distorsión morfológica del riñón izquierdo con gran componente graso en su polo superior que provoca compresión y desplazamiento anterosuperior del bazo y medial del resto de las estructuras intra-abdominales, así como en seno renal y cara posterior del riñón. Como otro signo de afectación visceral observemos el nódulo hepático (Fig. 4).

En la pielografía intravenosa se constata que el riñón izquierdo no es funcionante por lo que se 


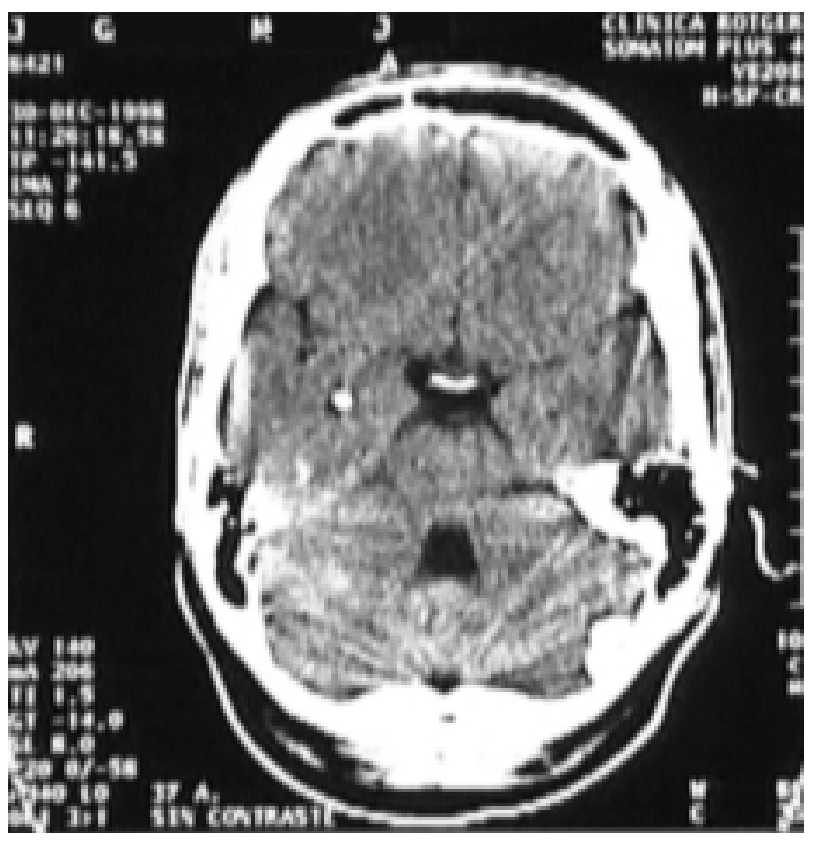

FIGURA 3. Hamartomas calcificados hemisferio cerebeloso izquierdo.

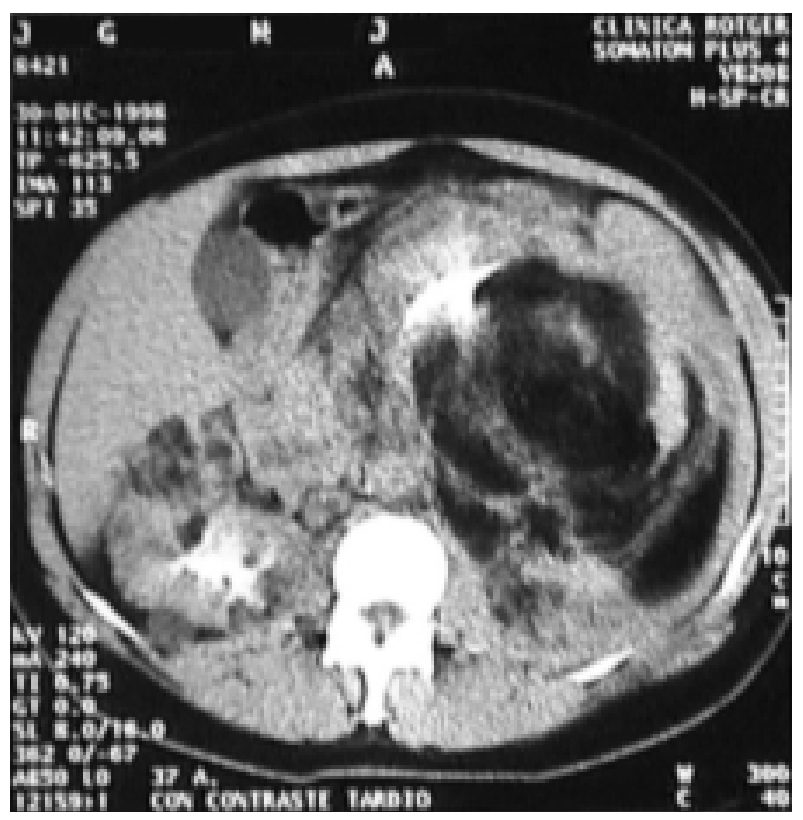

FIGURA 4. Hamartomas viscerales en ambos riñones $e$ higado.

decide cirugía de exéresis izquierda y linfadenectomía el 29 de enero de 1999. Extrayéndose un riñón cuyo peso inmediato fue de $6.300 \mathrm{~g}$. A través de una vía de abordaje anterior.

El 5 de febrero de 1999 se recibe informe de la pieza extraída: 31 x $19 \mathrm{~cm}$ y 5.235 g de peso. Tumoración de contornos lobulados, de $27 \mathrm{~cm}$ de diámetro de macroscopía polimorfa, alternando áreas parduzcas con otras amarillentas de aspecto graso. Se identifica riñón residual comprimido de $14 \times 5,5 \mathrm{~cm}$ de diámetro que incluye otra tumoración nodular, bien delimitada, gris homogénea de $3,5 \mathrm{~cm}$. Se aíslan 4 ganglios linfáticos; el mayor de 2,8 cm. Microscópicamente las dos tumoraciones están integradas por una proliferación celular en la que a todos los niveles se entremezclan tejido adiposo maduro, vasos irregulares de pared tortuosa y celularidad mesenquimal de músculo liso. Esta última predomina observándose zonas fasciculadas junto a otras poligonales, con frecuente multinucleación y ocasionales elementos abigarrados no anaplásicos. La tumoración es de límites infiltrativos extendiéndose al parénquima renal adyacente y grasa. El tejido renal se muestra con esclerosis atrófica por compresión. Ganglios linfáticos sin infiltración tumoral. No se han identificado signos morfológicos de malignidad. Se concluye: angiomiolipoma renal en dos tumoraciones de 27 y $3,5 \mathrm{~cm}$ de diámetro mayor, ausencia de metástasis ganglionares.

El 6 de marzo de 2001 presenta HTA 170/110, visto por Cardiología presenta insuficiencia mitral y aórtica mínima.

El 2 de abril de 2001 se realiza pielografía intravenosa que muestra riñón derecho grande con angiomiolipomas (Fig. 5).

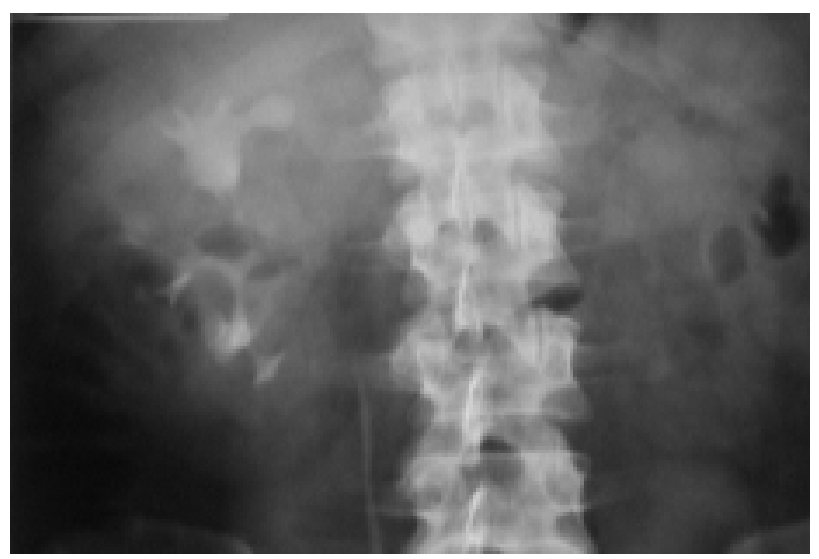

FIGURA 5. Vista de la pielografia.

Hace régimen y se trata la HTA con Medicina Interna: tenormin y astudal.

El 17 de abril de 2002 se realiza TAC: angiomiolipomas múltiples (Fig. 6). 


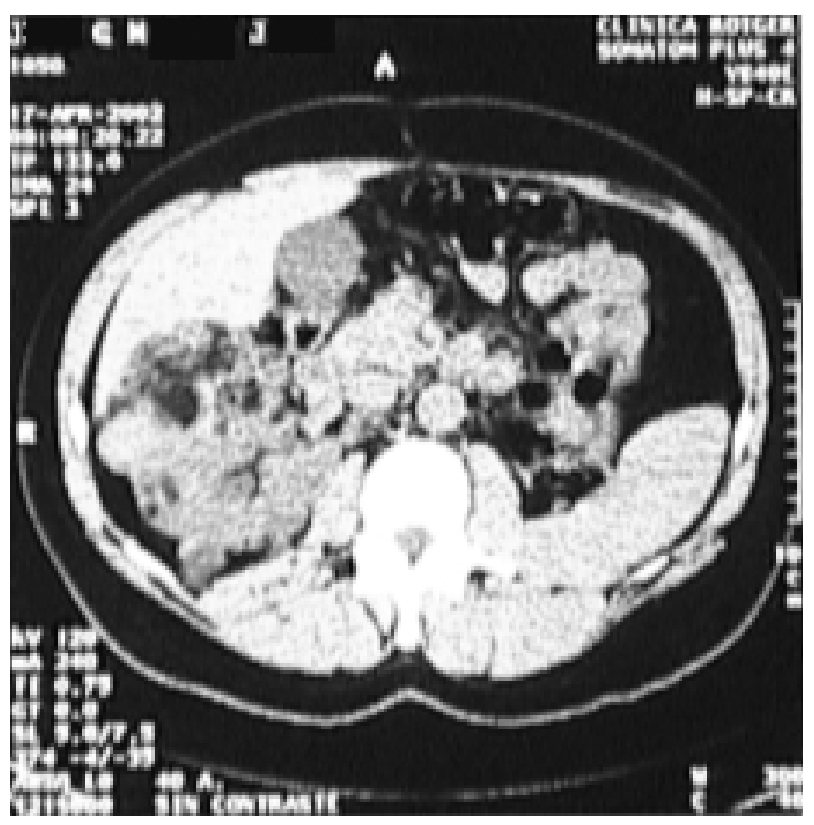

FIGURA 6. Hamartomas residuales post-operatorios.

Actualmente se encuentra controlado de la hipertensión arterial, con buen estado de salud.

\section{DISCUSIÓN}

El AML es el tumor mesenquimatoso más común del riñón ${ }^{1}$. Cuando aparece aislado de las facomatosis son únicos, frecuentemente asintomáticos, tienen una mayor frecuencia en el sexo femenino y son típicos de la quinta o sexta década de la vida ${ }^{3}$. Cuando aparece asociado a las facomatosis lo hace con mayor frecuencia a la Esclerosis Tuberosa o Enfermedad de Bourneville en un 40 a $80 \%$ de $\operatorname{los} \operatorname{casos}^{5}$, como lo es nuestro paciente, entonces los AML son frecuentemente múltiples, bilaterales, sintomáticos y sin predominancia de sexo. Cursa con crisis epilépticas, retraso mental, estigmas cutáneos característicos y anomalías en riñón, corazón, pulmón y otros órganos ${ }^{6}$. En nuestro caso encontramos los estigmas cutáneos, la presencia de hamartomas renales bilaterales, en hígado, cerebro y cerebelo. No hay manifestaciones epilépticas ni de retraso mental. Oesterling ${ }^{7}$ demostró que el $94 \%$ de las lesiones sintomáticas tenían al menos $4 \mathrm{~cm}$ de diámetro, mientras que el $64 \%$ de los asintomáticos eran menores de esa medida. Sin embargo en nuestro paciente no había sido descubierto el tumor hasta que no es estudiado por el traumatismo que sufrió. Se mantuvo asintomático a pesar de sobrepasar ampliamente las medidas descritas, con un AML gigante de más de $6 \mathrm{~kg}$. Aunque las lesiones características cutáneas de la Esclerosis Tuberosa pudieron haber sugerido la enfermedad, no ocurre así por haber sido interpretadas como una acné común. Nuestro paciente no presentó los síntomas comunes al AML como son: dolor, hematuria importante, anemia e hipertensión ${ }^{8}$.

El gran tamaño del tumor renal izquierdo así como la afectación del parénquima funcionante del mismo condicionaron la realización de la nefrectomía ${ }^{5}$. En la pieza no se encontró tumor maligno ni infiltración ganglionar. Se conviene en recomendar el tratamiento de aquellos tumores mayores de $4 \mathrm{~cm}$ de diámetro ya que evolucionan con mayor frecuencia a la aparición de síntomas y a las complicaciones hemorrágicas ${ }^{9}$. En nuestro caso no se determinó la existencia de hemorragia en su evolución a pesar de su gran tamaño; la infección urinaria fue la única manifestación atribuible al tumor.

Entre las diversas modalidades terapéuticas se encuentran: nefrectomía parcial, embolización del tumor, nefrectomía total o radical y crioterapia $^{10}$. En general el tratamiento se condicionará por:

1. El tamaño del tumor.

2. La reserva funcional renal en el caso de tumores bilaterales o riñones únicos ${ }^{16}$.

3. La existencia de un crecimiento activo en el seguimiento ecográfico ${ }^{6}$.

4. La duda diagnóstica ${ }^{3}$ diferencial con tumores malignos, ante la cual preferimos la exéresis de la víscera sobre la punción aspirativa diagnóstica ${ }^{11}$. La posibilidad de una variante epitelioide del AML de carácter maligno refuerza esta conducta. Esta variante se caracteriza por la presencia de células epitelioides y una alta reactividad al marcador específico de melanoma HMB-45 considerado difícil de diagnosticar excepto por cirugía o autopsia ${ }^{12}$.

5. La aparición de síntomas o complicaciones como la hemorragia retroperitoneal espontánea ${ }^{13}$.

6. En la mujer, la prevención de posibles complicaciones graves en el embarazo o el parto $^{14,15}$. 
En el caso que presentamos se realizó la nefrectomía por la carencia de valor funcional en el riñón izquierdo, presentar un gran tamaño y la aparición de síntomas después del traumatismo.

\section{REFERENCIAS}

1. KWAI-FONG NG, TSE-CHING CHEN.: Infiltrating renal angiomyolipoma into ascending colon associated with hepatic involvement in a patient with tuberous sclerosis. J Urol 2001 jan; 165: 179.

2. KANDEMIR O, TATLISEN A, KONTAS O, ÖRSKIRAN G, KAHYA H.: Sarcomatoid squamous cell carcinoma of the right renal pelvis with liver metastasis: case report. J Urol 1995 jun; 153: 1895-1896.

3. DE LUCA S, TERRONE C, ROCCA ROSSETTI S.: Management of renal angiomyolipoma: report of 53 cases. BJU Int $1999 \mathrm{feb}$; 83: 215-218.

4. STEDMAN TL.: Stedman's Medical Dictionary. 26th ed, Baltimore: Williams \& Wilkins 1995; 760: 86.

5. STEINER MS, GOLDMAN SM, FISHMAN EK y cols.: The natural history of renal angiomyolipoma. J Urol 1993; 150: 1792.

6. VERDÚ TARTAJO F, HERRANZ AMO F, SUBIRÁ RÍOS D.: Angiomiolipoma renal. En: Herranz Amo F. Tumores del riñón. $1^{\mathfrak{a}}$ ed. Ediciones Doyma, S.L. 2001: 241-245.

7. OESTERLING JE, FISHMAN EK, GOLGMAN SM, MARSHALL FF.: The management of renal angiomyolipoma. J Urol 1986; 135: 1121.

8. FAZELI-MATIN S, NOVIC AC.: Nephron-sparing surgery for renal angiomyolipoma. Urology 1998; 52: 577.

9. CLARK EP, NOVICK AC.: Exophytic noninvasive growth pattern of renal angiomyolipomas: implications for nephron sparing surgery. J Urol 2001 feb; 165: $513-514$
10. DELWORTH MG, PISTERS LL, FORNAGE BD, ESCHENBACH AC.: Cryotherapy for renal cell carcinoma and angiomyolipoma. J Urol 1996 jan; 155: 252-255.

11. WADIH GE, RAAB SS, SILVERMAN F.: Fine needle aspiration citology of renal and retroperitoneal angiomyolipoma. Report of two cases with cytologic findings and clinicopathologic pitfalls in diagnosis. Acta Cytolog 1995; 39: 945.

12. YAMAMOTO $\mathrm{T}$, ITO $\mathrm{K}$, SUZUKI $\mathrm{K}$, YAMANAKA $\mathrm{H}$, EBIHARA K, SASAKI A.: Rapidly progressive malignant epitheliod angiomyolipoma of the kidney. $J$ Urol 2002 jul; 168: 190-191.

13. RUIZ CERDÁ JL, JIMÉNEZ CRUZ JF.: Tumores del parénquima renal. En: Jiménez Cruz JF y Rioja Sanz LA editores. Tratado de Urología. Tomo II. Barcelona, España: J.R. Prous S.A. 1993: 1095-1097.

14. MARTÍNEZ M, CAMACHO F, MADRID JL y cols.: Hemorragia intraperitoneal tras parto como manifestación de un angiomiolipoma renal. Arch Esp de Urol 1989; 42: 25.

15. FERNÁNDEZ ARJONA M, MÍNGUEZ R, SERRANO y cols.: Angiomiolipoma renal de rápido crecimiento asociado a embarazo. Actas Urol Esp 1994; 18: 755.

16. BLUTE ML, MALEK RS, SEGURA JW.: Angiomyolipoma: clinical metamorphosis and concepts for management. J Urol 1998; 139: 20.

Dr. M. Rosselló Barbará

Centro de Urología, Andrología y Sexología

Clínica Rotger

C/ Santiago Russiñol, 9 - $3^{\text {a }}$ planta

07012 Palma de Mallorca (Baleares)

(Trabajo recibido el 9 enero de 2003) 